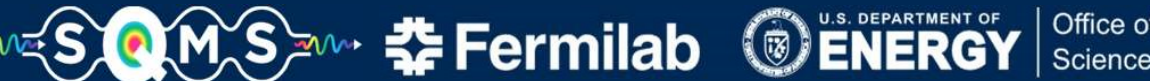

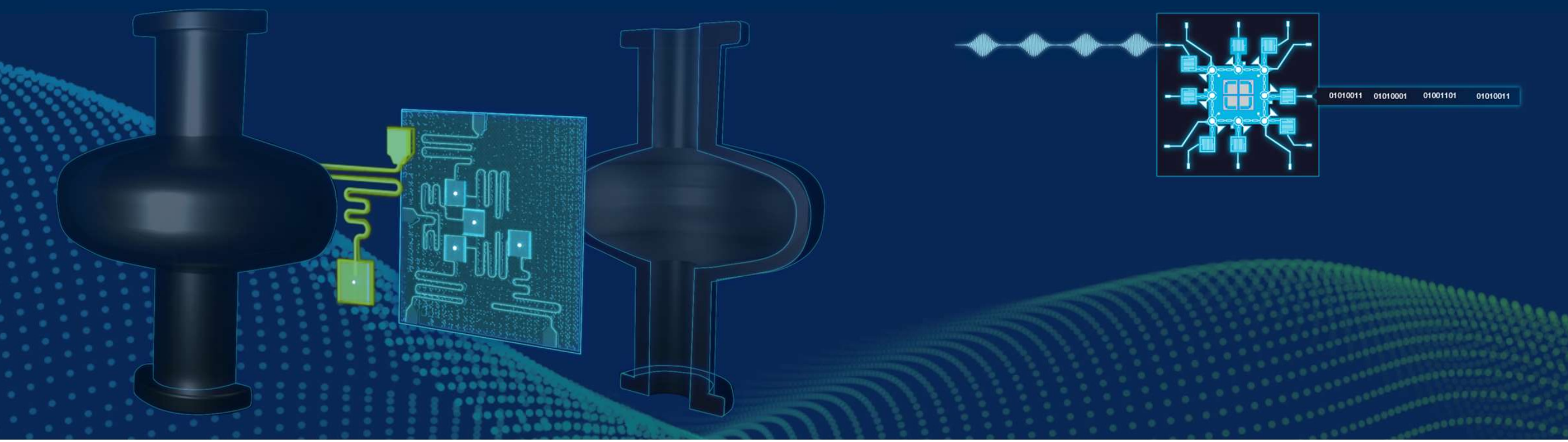

\section{Dilution Refrigerators for Quantum Science}

Matthew Hollister

ICMC Short Course: Quantum Information Science International Cryogenic Materials Conference 2021 


\section{Course outline and objectives}

Objective: To understand the physics behind the dilution cooling process, be familiar with the historical development and the design features of modern refrigerators.

\section{Outline:}

- Physics of helium and the behavior of $3 \mathrm{He} / 4 \mathrm{He}$ mixtures

- Making a practical refrigerator

- The development of dilution refrigerators from the 1960 s to today

- Modern designs and operational matters

- Future developments 


\section{Physics of Helium and Helium Mixtures}




\section{Helium mixtures}

- Important to note that Helium-4 obeys Bose statistics. Superfluid transition occurs at 2.18 K. Helium-3 obeys Fermi statistics, obeys the Pauli principle and has a superfluid transition near $1 \mathrm{mK}$.

- In the following discussions, the respective concentrations of the two helium isotopes are expressed as

$$
\begin{gathered}
x=x_{3}=\frac{n_{3}}{n_{3}+n_{4}} \\
x_{4}=\frac{n_{4}}{n_{3}+n_{4}}
\end{gathered}
$$

where $n_{3}$ and $n_{4}$ are the number of atoms or the number of moles of $3 \mathrm{He}$ and $4 \mathrm{He}$, respectively. 


\section{Phase separation at low temperature}

4He-rich phase

Higher density

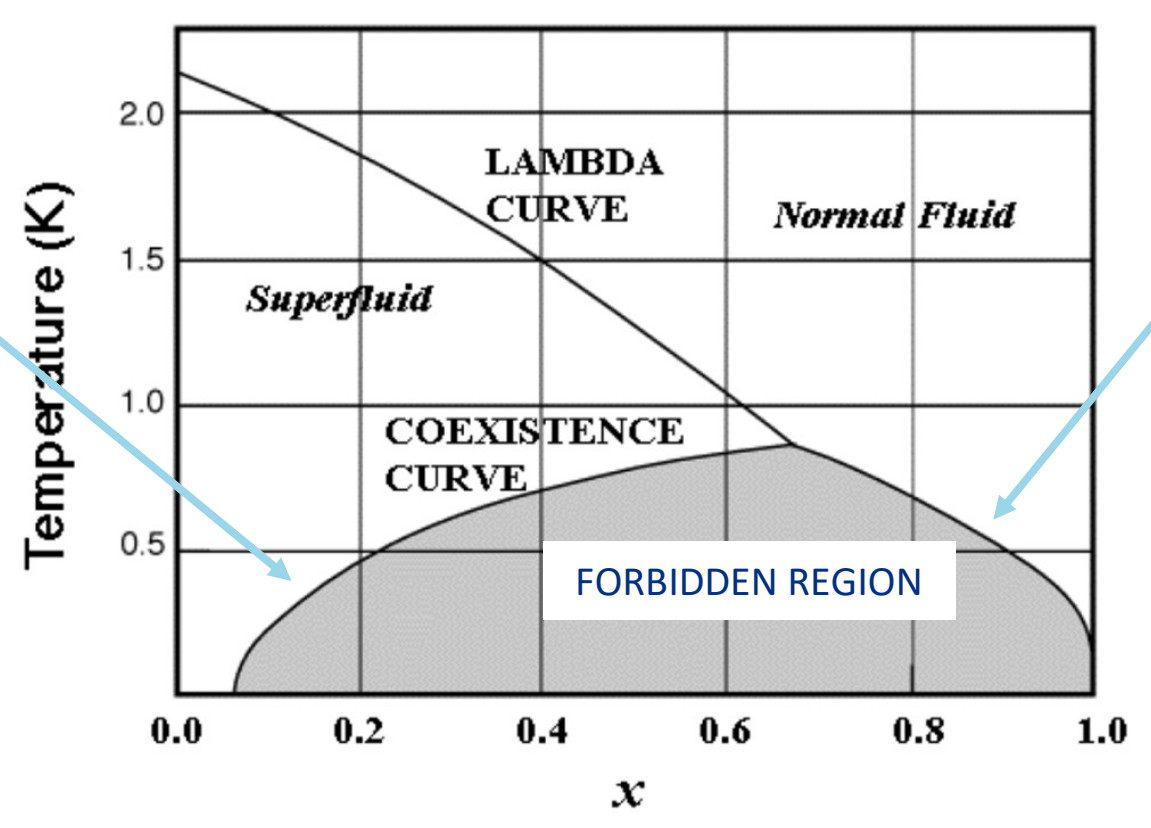

Hollister | Dilution Refrigerators for Quantum Science
3He-rich phase

(essentially pure $3 \mathrm{He}$ )

Lower density, floats on top of the $4 \mathrm{He}$-rich phase 


\section{He-4He mixtures as Fermi liquids}

- As mentioned, $4 \mathrm{He}$ obeys Bose statistics and will condense into the quantum mechanical ground state at low enough temperature.

- In a helium mixture, the condensed $4 \mathrm{He}$ acts as an "inert superfluid background" which contributes to the volume of the liquid and the effective mass of the $3 \mathrm{He}$ atoms but has zero heat capacity.

- The 3He obeys Fermi statistics and will behave in a similar way to conduction electrons in a metal. However, whereas conduction electrons have a Fermi temperature of $\sim 10000 \mathrm{~K}$, the Fermi temperature of the $3 \mathrm{He}$ is $\sim 1 \mathrm{~K}$. 


\section{Finite solubility (1)}

- Let us consider whether a $3 \mathrm{He}$ atom at the phase separation line would "prefer" to be in the $3 \mathrm{He}$ phase or be in the $4 \mathrm{He}$-rich phase. The $3 \mathrm{He}$ will go into the phase with the larger binding energy.

- For $\mathbf{3 H e}$ atoms in pure $\mathbf{3 H e}$, the binding energy is the latent heat of evaporation.

- For $3 \mathrm{He}$ atoms in $\mathbf{4 H e}$, although the van der Waals forces between $4 \mathrm{He}$ and $3 \mathrm{He}$ are identical, the $3 \mathrm{He}$ atoms have larger zero-point motion due to their smaller mass.

- The $4 \mathrm{He}$ atoms occupy a smaller volume than the $3 \mathrm{He}$ atoms, so $3 \mathrm{He}$ atoms will feel a stronger attraction to a $4 \mathrm{He}$ atom. 


\section{Finite solubility (2)}

- As more $3 \mathrm{He}$ atoms enter the $4 \mathrm{He}$ phase, two effects occur:

- The larger zero-point motion of the $3 \mathrm{He}$ means that the liquid near the $3 \mathrm{He}$ atoms is more dilute than near $4 \mathrm{He}$ atoms, and the $3 \mathrm{He}$ atoms will tend to group. Binding energy of the $3 \mathrm{He}$ atoms will increase with increasing $x_{3}$.

- The $3 \mathrm{He}$ atoms must obey the Pauli principle, so additional 3He atoms must occupy successively higher energy states. Binding energy of the $3 \mathrm{He}$ atoms will decrease with increasing $x_{3}$.

- The first effect is dominant - as $x_{3}$ increases, the binding energy of $3 \mathrm{He}$ in $4 \mathrm{He}$ approaches the binding energy of $3 \mathrm{He}$ in $3 \mathrm{He}$, and an equilibrium is reached.

- This is true even at $T=0$, hence the finite solubility effect. 


\section{Cooling power of the dilution refrigerator}

- Measurements of the specific heat shows that the enthalpy of $3 \mathrm{He}$ in the dilute phase is larger than in the concentrated phase. Moving $3 \mathrm{He}$ from the concentrated to the dilute phase will result in cooling due to the heat of mixing

$$
\dot{Q}(T)=\dot{n}_{3}\left(H_{d}(T)-H_{c}(T)\right)
$$

- We won't work through the full derivation here, but in summary

$$
\begin{gathered}
H_{3}(T)=H_{3}(0)+11 T^{2} \\
H_{3, d}(T)=H_{3}(0)+95 T^{2} \\
\dot{Q}(T)=84 \dot{n}_{3} T^{2}
\end{gathered}
$$

This result is correct to with a few $\%$ for $T<40 \mathrm{mK}$ 


\section{Cooling power - things to note}

- The equation giving the cooling power is for the liquid in the mixing chamber. It takes no account of the Kaptiza resistance between the liquid and the chamber itself, or the contact resistance between the chamber and any external equipment such as a cold plate.

- Derivation assumes that the $3 \mathrm{He}$ in the concentrated and dilute phases are at the same temperature. In reality, the precooling of the inflowing $3 \mathrm{He}$ won't be perfect, so we modify the derviation to account to $T_{N}$, the temperature of the 3 He leaving the last heat exchanger:

$$
\dot{Q}=\dot{n}_{3}\left(96 T_{m c}^{2}-12 T_{N}^{2}\right)
$$




\section{Osmotic pressure}

- The osmotic pressure of the Helium mixture is a function of temperature and $3 \mathrm{He}$ concentration (see plot right from Betts, 1989).

- We will discuss this in more detail in the next section, but if we pump 3He away from the dilute phase the concentration will be reduced, resulting in a difference in the osmotic pressure and driving the flow of $3 \mathrm{He}$.

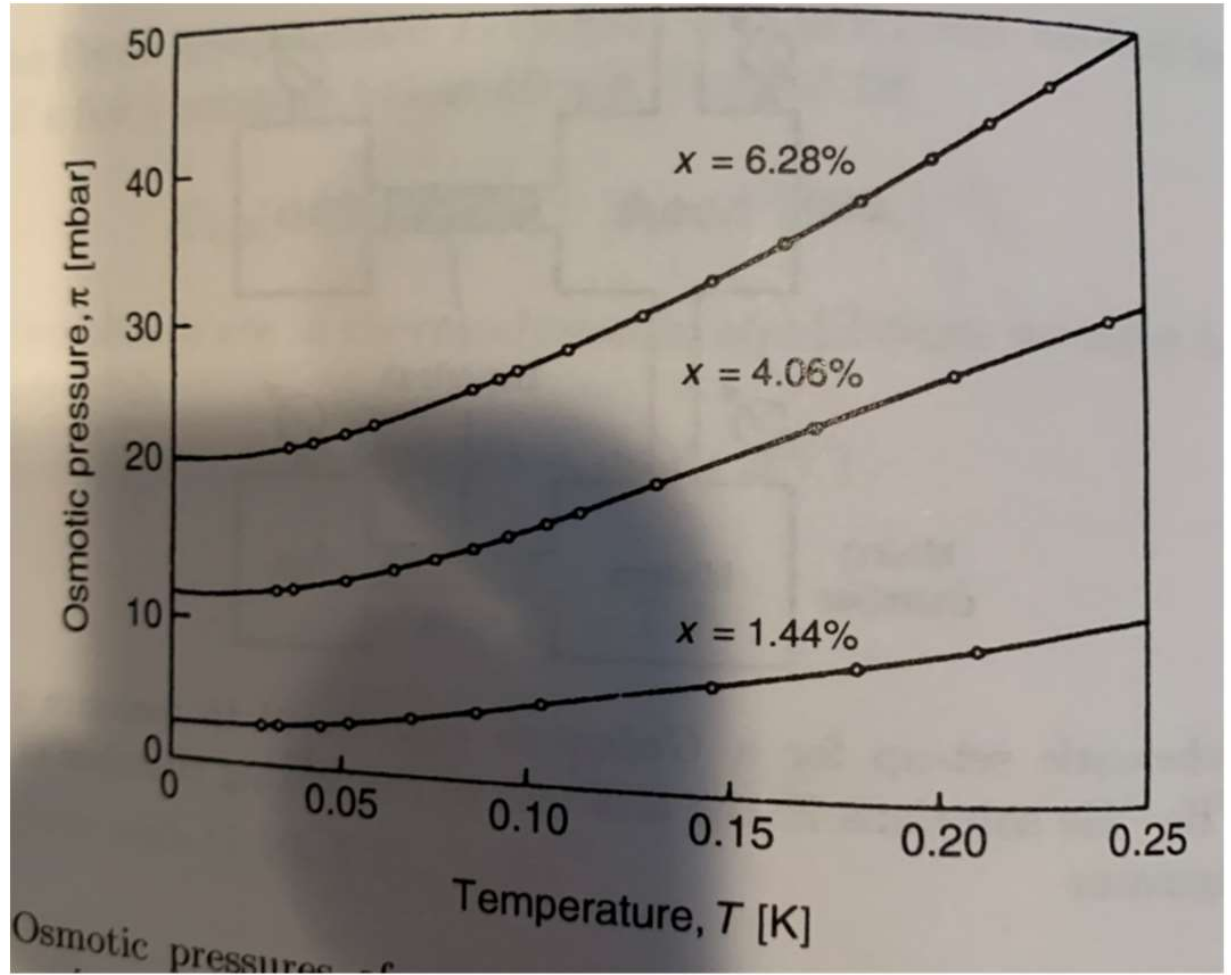




\section{Building a Refrigerator}




\section{General layout of a dilution refrigerator}

- Simplest model for the refrigerator is a U-tube, with the concentrated phase on one side and the dilute side on the other.

- As observed previously, the less dense concentrated phase floats on top of the dilute phase since the superfluid has much higher density.

- Removing $3 \mathrm{He}$ from the dilute phase surface upsets the equilibrium.

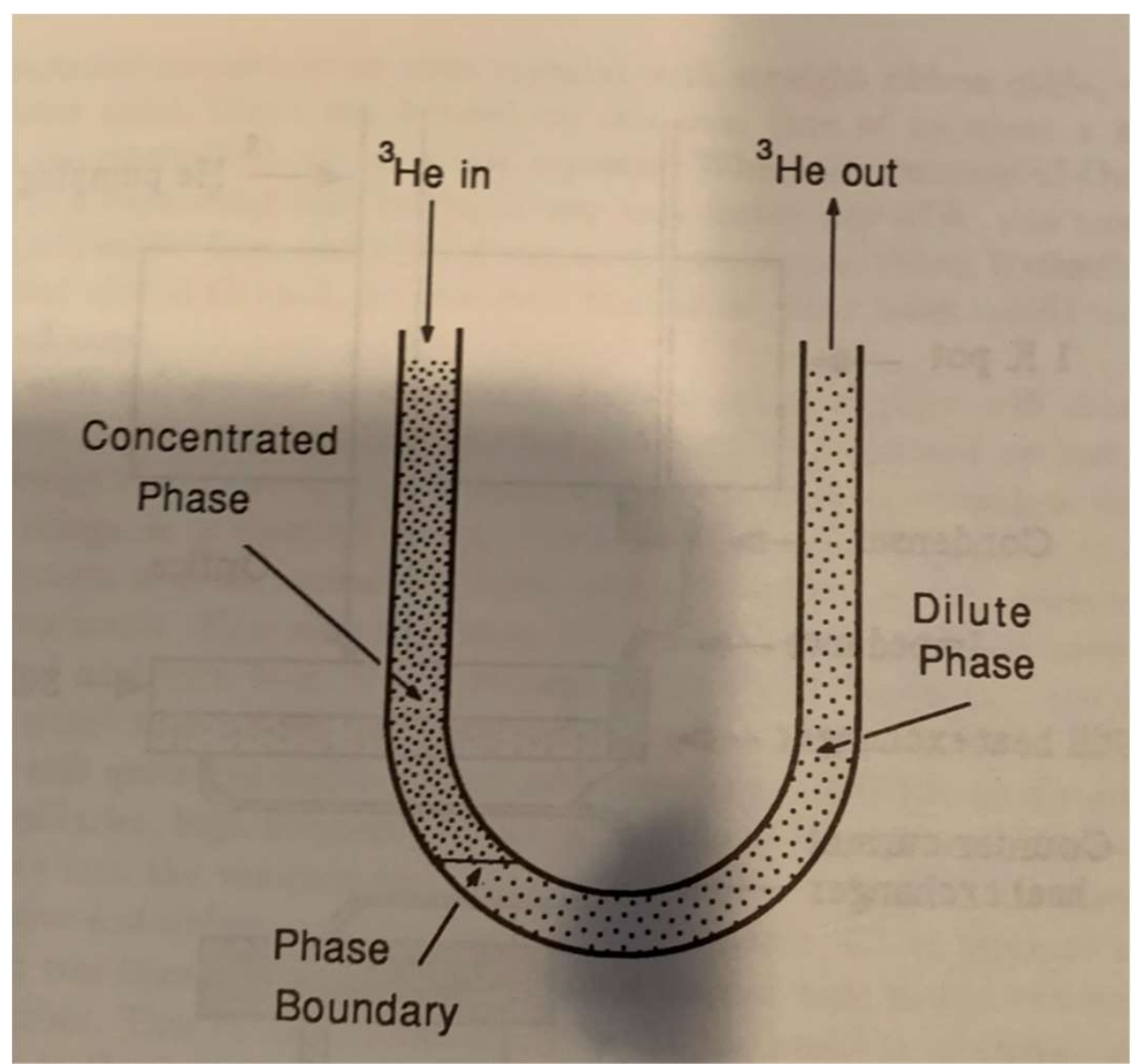




\section{General refrigerator layout}

- Major components of a generalized dilution refrigerator shown in the diagram.

- 3 He from the room temperature pump system is cooled and liquified at/above the still

- Cooled further on the way to the mixing chamber

- $3 \mathrm{He}$ is pumped away from the still

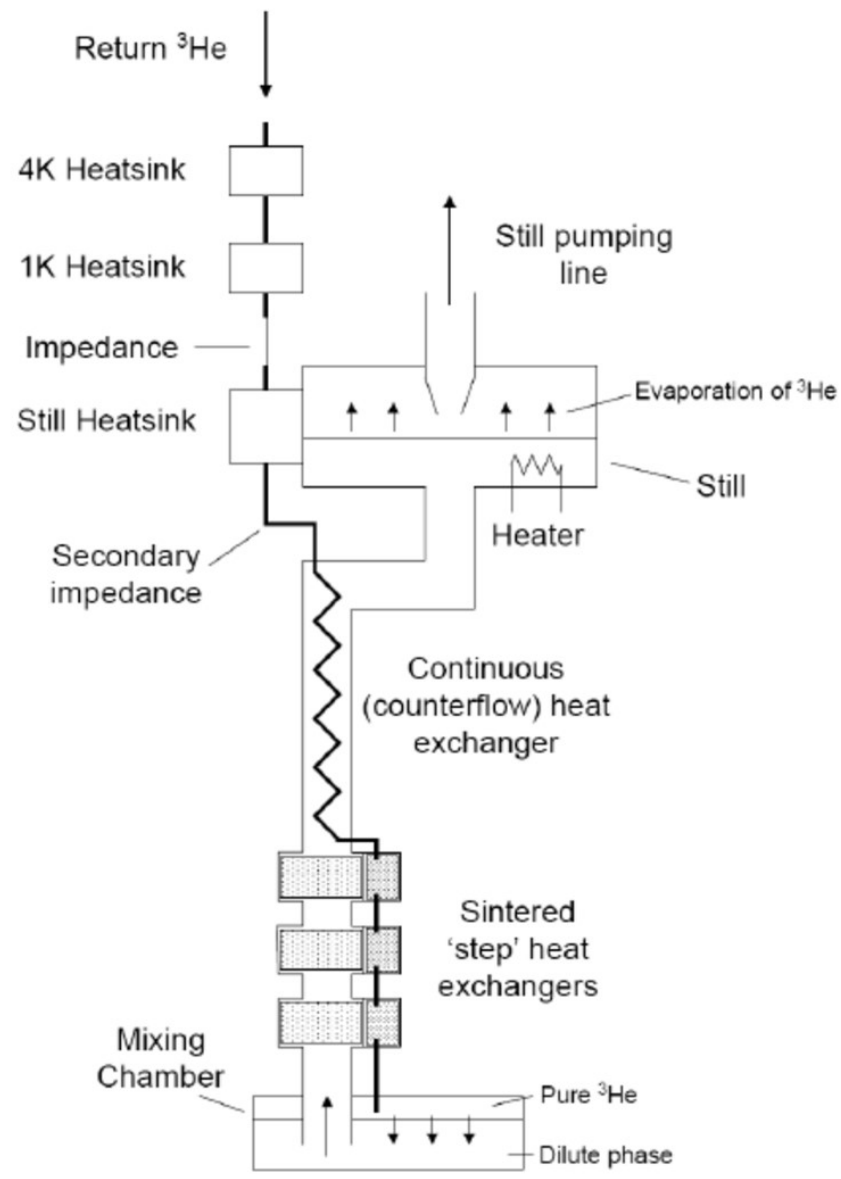




\section{Heat exchanger design above $50 \mathrm{mK}$}

- At higher temperatures, continuous heat exchangers work well. These are typically of the concentric (or coil-intube) configuration.

- Heat is transferred from the dilute phase in the annular space and the concentrated phase in the coiled capillary.

- Heat transfer along the fluid and the capillary are negligible.

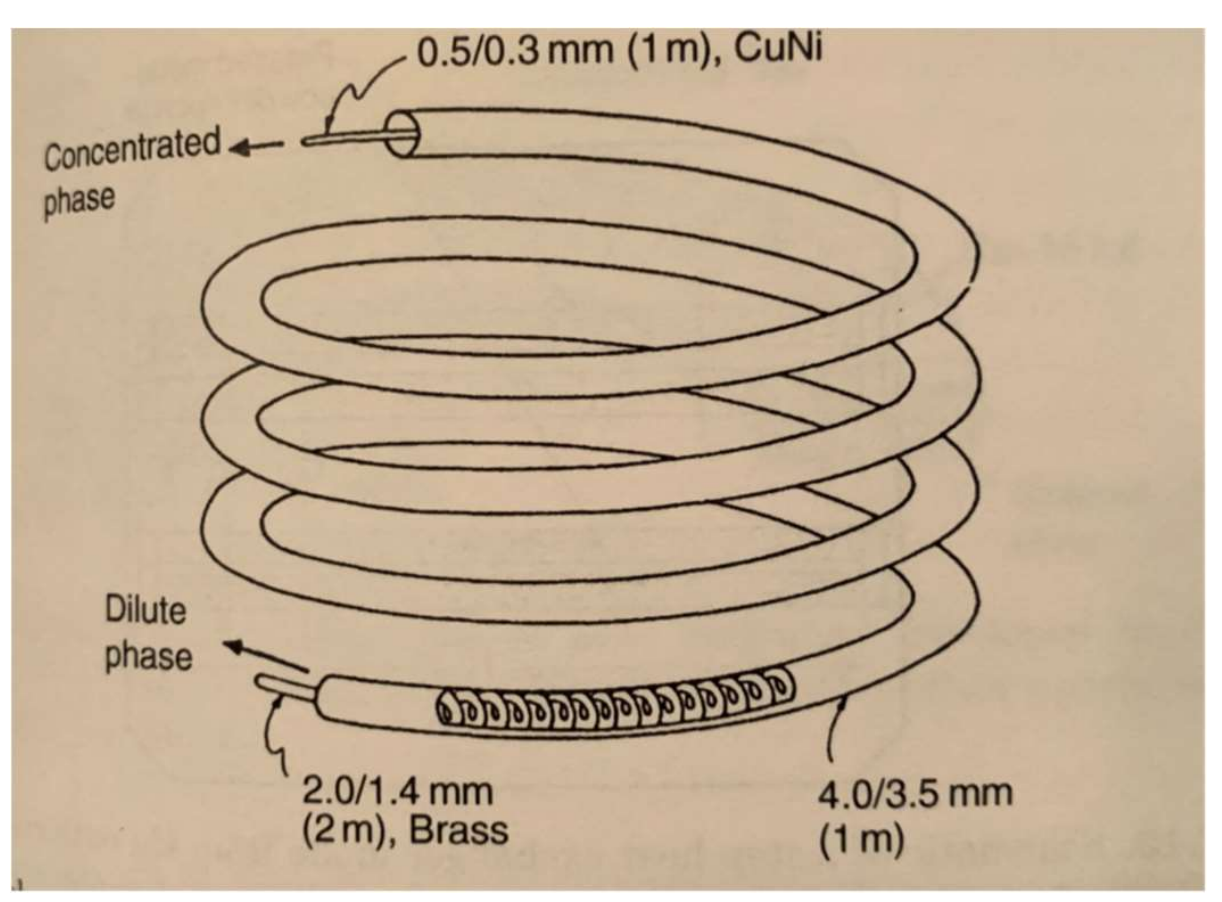




\section{Heat exchanger design below $50 \mathrm{mK}$}

- Designs using continuous heat exchangers can reach $\sim 30 \mathrm{mK}$.

- For lower temperatures or very high flow rates, larger surface area heat exchangers are needed to overcome the increasing Kaptiza resistance.

- Simple form is a sintered metal block to

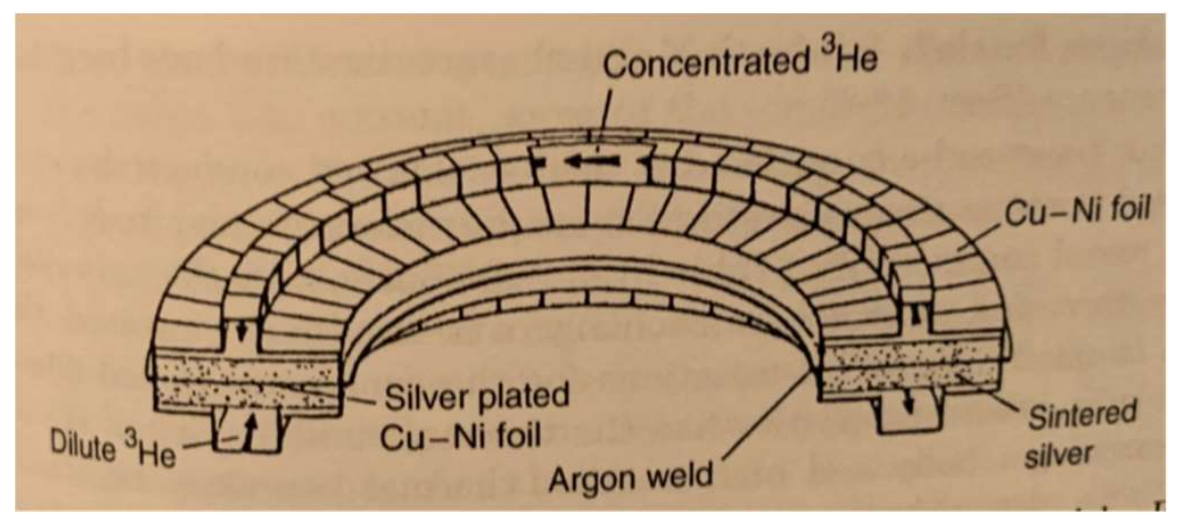
give large surface area, with a machined channel to reduce flow impedance (and hence viscous heating).

- To minimize axial heat flow, several discrete exchangers are connected in series - "step" heat exchangers. 


\section{Practical configuration example}

- The diameter of the flow channels

increases as the temperature is lowered due to the decreasing density.

- Dilute channel has larger diameter than the concentrated side due to the lower density.

\begin{tabular}{lllll}
\hline Exchanger No & Length & Area & $\begin{array}{l}\text { Dilute side } \\
\text { channel size }\end{array}$ & $\begin{array}{l}\text { Concentrated } \\
\text { channel size }\end{array}$ \\
\hline Tubular & $\begin{array}{l}9 \mathrm{~m} \text { coiled } \phi 3 \mathrm{~mm} \\
\text { tube inside } 4 \mathrm{~m} \\
\text { of } \phi 5 \mathrm{~mm} \text { tube }\end{array}$ & $0.08 \mathrm{~m}^{2}$ & $\begin{array}{l}\phi 2.5 \mathrm{~mm} \text { (clear } \\
\text { bore inside coil) }\end{array}$ & $\begin{array}{l}\text { not well-defined } \\
\text { (flattened tube) }\end{array}$ \\
\hline 0 (above $20 \mathrm{mK}$ flange) & $60 \mathrm{~mm}$ & $24 \mathrm{~m}^{2 *}$ & $3 \mathrm{~mm}$ & $2 \mathrm{~mm}$ \\
1 & $60 \mathrm{~mm}$ & $24 \mathrm{~m}^{2 *}$ & $3 \mathrm{~mm}$ & $2 \mathrm{~mm}$ \\
2 & $60 \mathrm{~mm}$ & $24 \mathrm{~m}^{2 *}$ & $3 \mathrm{~mm}$ & $2 \mathrm{~mm}$ \\
3 & $60 \mathrm{~mm}$ & $24 \mathrm{~m}^{2 *}$ & $4 \mathrm{~mm}$ & $3 \mathrm{~mm}$ \\
4 & $60 \mathrm{~mm}$ & $24 \mathrm{~m}^{2 *}$ & $4 \mathrm{~mm}$ & $3 \mathrm{~mm}$ \\
5 & $720 \mathrm{~mm}$ & $30 \mathrm{~m}^{2 *}$ & $7 \mathrm{~mm}$ & $4 \mathrm{~mm}$ \\
6 & $720 \mathrm{~mm}$ & $60 \mathrm{~m}^{2 *}$ & $9 \mathrm{~mm}$ & $4 \mathrm{~mm}$ \\
7 & $720 \mathrm{~mm}$ & $60 \mathrm{~m}^{2 *}$ & $11 \mathrm{~mm}$ & $5 \mathrm{~mm}$ \\
8 & $720 \mathrm{~mm}$ & $70 \mathrm{~m}^{2 *}$ & $13 \mathrm{~mm}$ & $5 \mathrm{~mm}$ \\
\hline *-estimate & & &
\end{tabular}

Table from Bradley et al. (1982). Fridge designed to operate at $3 \mathrm{mK}$ 


\section{Mixing chamber}

- The mixing chamber is a relatively simple. Major requirement is for a large contact area between the helium phases and the metal.

- This is typically achieved through sintered structures, such as the silver sponge example shown right.

- In this design, the diameter of the mixing chamber is approximately $100 \mathrm{~mm}$ and each sponge has a surface area of $10 \mathrm{~m}^{2}$.

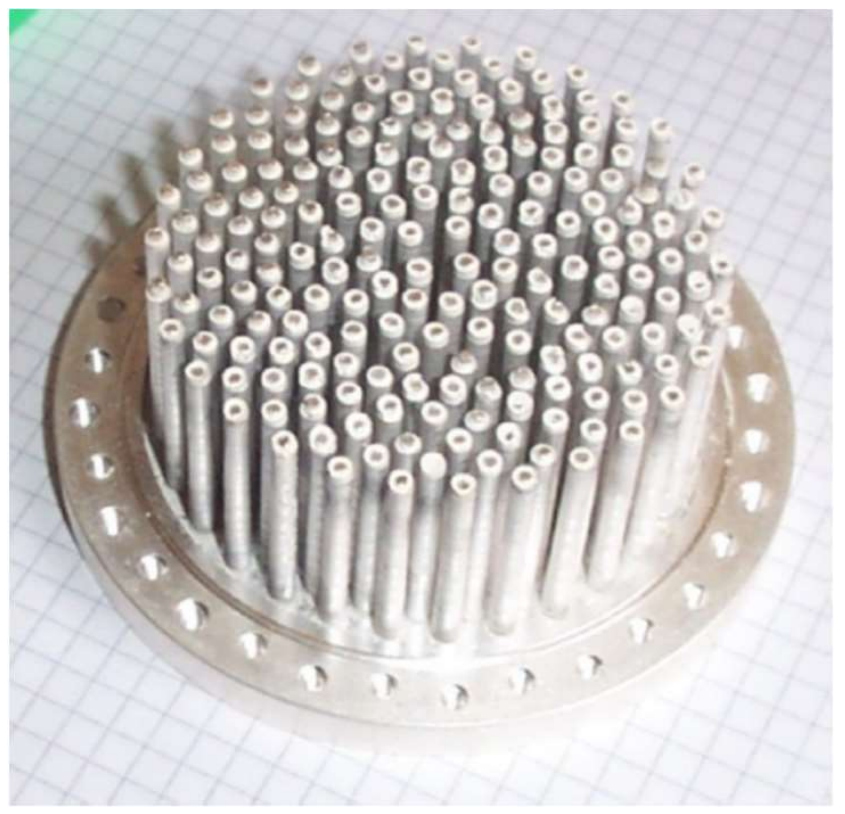

Mixing chamber construction example from Uhlig (2015) 


\section{Room temperature components - Pumps}

- Choice of pumps depend on circulation rate and desired base temperature.

- Usually consist of a primary pump (scroll or roots).

- Still pumping may be supplemented with one or more turbomolecular pumps for higher-performance systems.

- For efficient condensation, it is often necessary to use a compressor to increase the inlet pressure (and decrease the outlet pressure of the primary pump).

- Important that pumps are sealed for helium use and do not contaminate the process line. 


\section{Room temperature components - Filtering and sealing}

- Majority of the gas handling at room temperature will be subatmospheric, so will tend to suck in air that could contaminate the process line.

- Fridges generally include cold filters with Pt or other metal sinters internally

- Many will also include an external activated charcoal trap in liquid nitrogen

- For very long duration runs, an LHe or 4-K trap can also be used.

- Choice of flanges and seals is important. Metal seals generally much better than rubber, but harder to work with.

- Be wary of parts of the system that will see internal pressure. If using rubber Orings, it may be necessary to add overpressure rings. 


\section{History and Development of the Dilution Refrigerator}




\section{Early commercialization}

- The first commercial refrigerators were built in the mid-1960s by Oxford instruments, first in collaboration with Heinz London, and later based on Hall's design licensed from the UK Atomic Energy Authority.

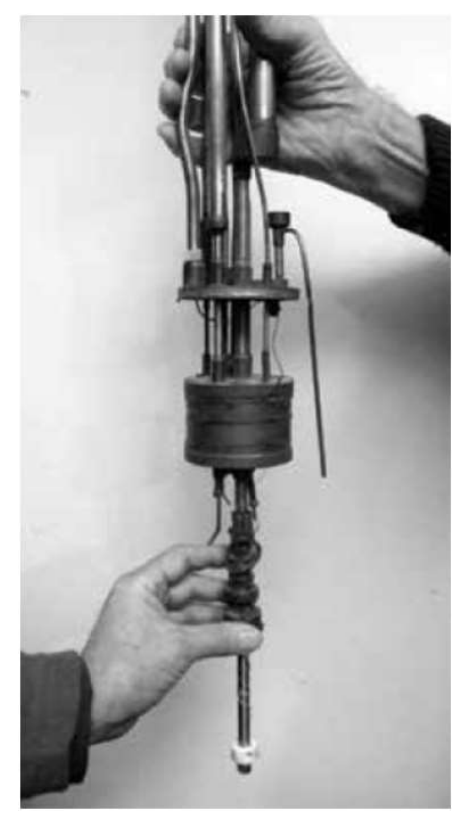

(Left) Early commercial refrigerator insert

(Right) Commercial refrigerator with copper heat exchangers, c.1975

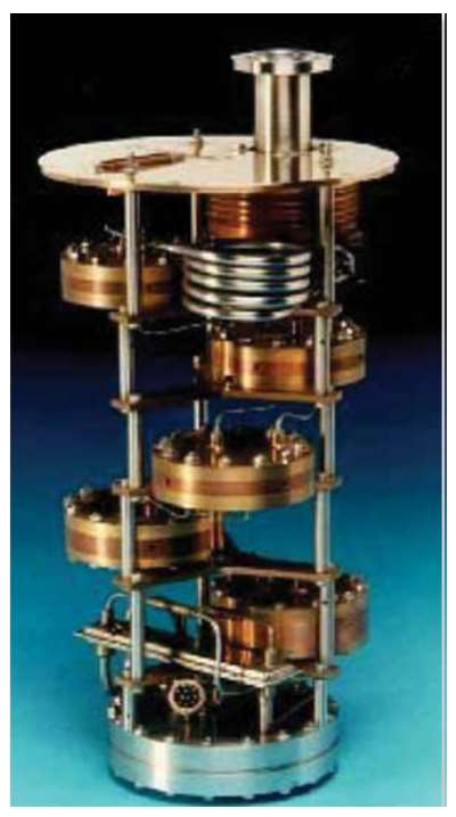




\section{Eliminating the liquid helium}

- The primary feature that distinguishes modern refrigerators from the older style is the use of mechanical precoolers in place of the liquid helium bath.

- This was made possible by an earlier innovation, the Joule-Thomson Heat Exchanger.

- Described independently by Kraus at ZTB and by de Waele at Eindhoven in 1977, this device eliminates the 1-K Pot in a wet dilution refrigerator by using a long counterflow exchanger cool the Helium-3 stream with gas pumped from the still, followed by an expansion valve.

- This stage cools and partially liquifies the Helium-3, with the remaining liquification taken care of by the still. 


\section{Eliminating the liquid helium (2)}

- Removing the 1-K Pot served three important purposes:

- The pumped pot can be a significant source of vibration in sensitive experiments

- The pumped pot can dominate the Helium consumption of a system

- The pot is prone to blockage, making it a common source of fridge failure

- Note that it would not be ideal to just cool and liquify the Helium-3 by running the gas directly to the still since the enthalpy of the gas is larger than that of the gas leaving the still at $\sim 1 \mathrm{~K}$.

- This has been demonstrated, but only for very low flow rate fridges with efficient precooling, so not a very practical approach. 


\section{Eliminating the liquid helium (3)}

- With improvements in mechanical cryocoolers in the late 1980s, it was a natural step to couple one to a dilution refrigerator.

- First example described by Pari in 1990 , followed by the pioneering development work of Uhlig starting in 1993.

- Pari's machine (shown right) used a Stirling cycle cooler operating at $12 \mathrm{~K}$ combined with a Helium-4 J-T to produce a temperature at $\sim 4 \mathrm{~K}$.

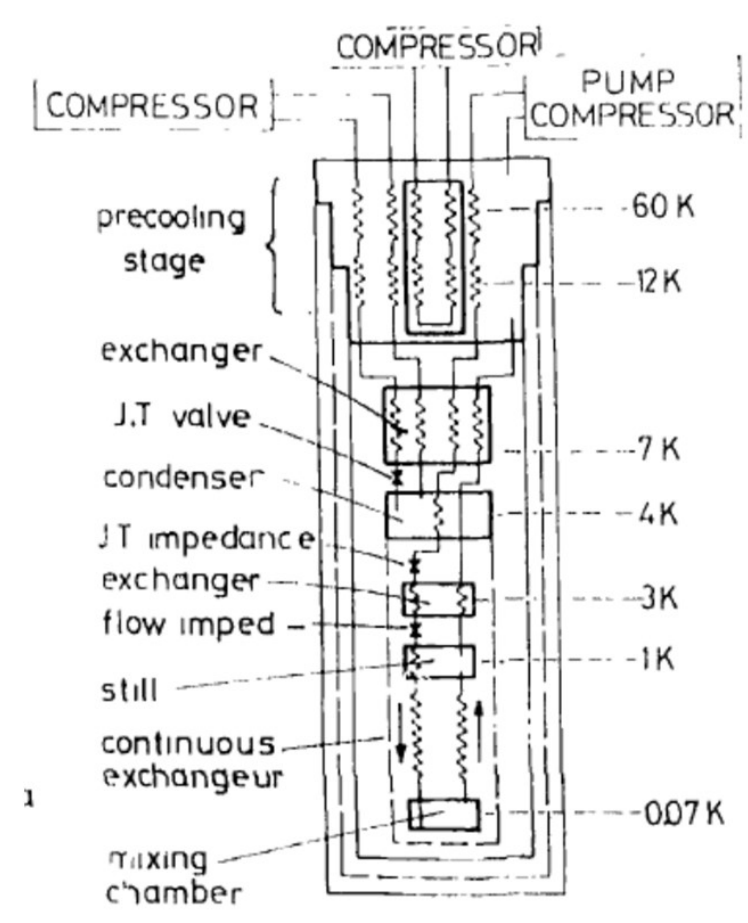




\section{Eliminating the liquid helium (4)}

- The development of Uhlig's group eliminated the Helium$4 \mathrm{~J}$-T stage, relying instead on the Joule Thompson Heat Exchangers used previously in wet refrigerators.

- These fridges (example shown right) initially used $10 \mathrm{~K}$ Gifford-McMahon coolers, and later small $4 \mathrm{~K}$ pulse tube coolers.

- Important to note that 4-K class cryocoolers were not available until 1999-2000. Much of the early work was carried out with $10-\mathrm{K}$ coolers.

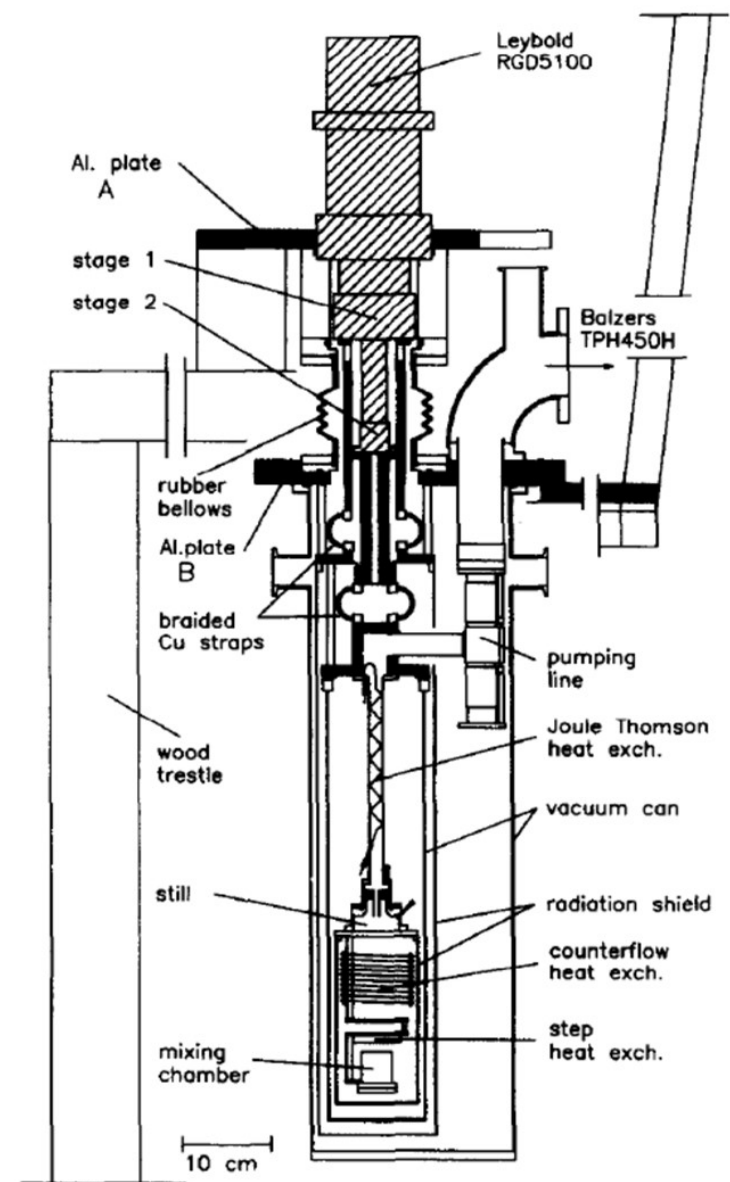




\section{“Dry" vs "Wet” Refrigerators}

Cooled by Pulse Tube to $\sim 50 \mathrm{~K}$

Cooled by Pulse Tube to $\sim 4 \mathrm{~K}$

$\sqrt{7}$

Cooled and partially liquified by JT

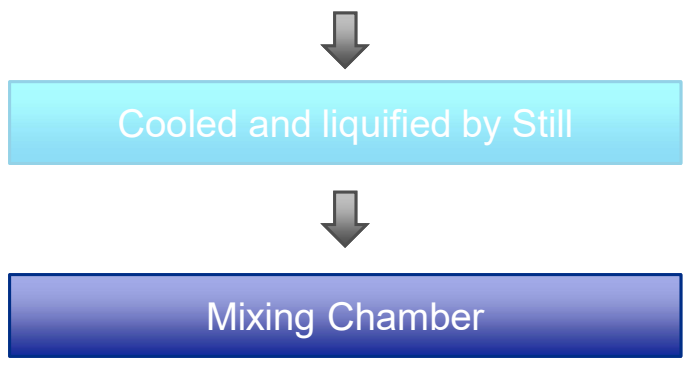

"Dry" Process
Cooled by Liquid Helium to $\sim 4 \mathrm{~K}$

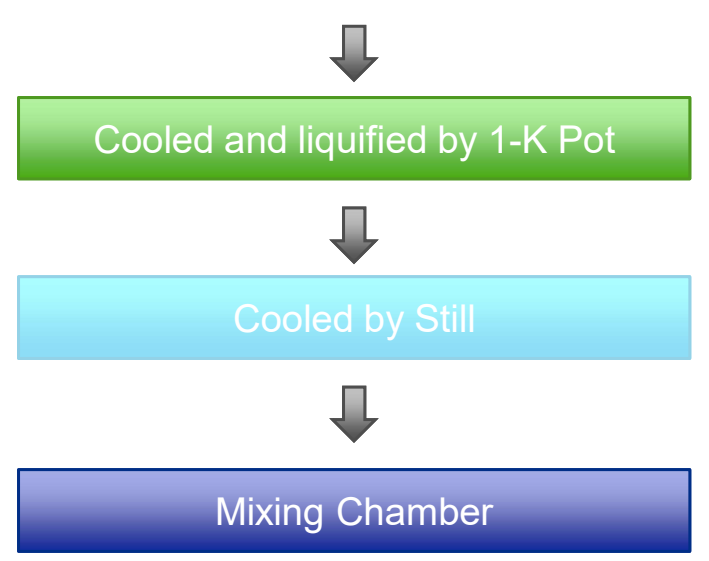

"Wet" Process 


\section{Early commercial dry fridges}

- Early examples of commercial cryogen-free fridges came from Leiden Cryogenics and Air Liquide in the early 2000s

- The Leiden refrigerator, built for the SCUBA-2 astronomical camera (right) is still operational at 14000 feet at the James Clerk Maxwell Telescope in Hawaii.

- Limitations of these early designs were often poor heat exchange at $4 \mathrm{~K}$ on the second stage of the pulse tube cooler.

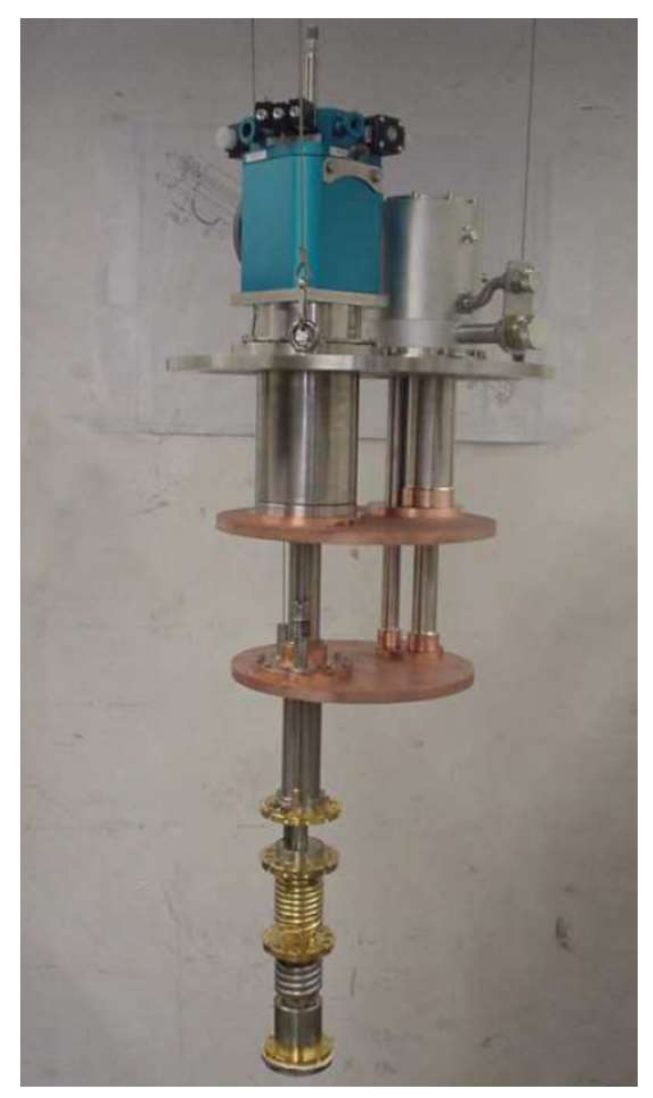




\section{Improvements to the precooler design}

- An interesting property of the pulse tube cooler was measured in the early 2000s in that excess cooling capacity on the regenerator between the two stages exists.

- Some heat can be dissipated on the regenerator with little impact on the $4 \mathrm{~K}$ cooling performance.

- Adding a coil to the regenerator provides a useful additional heat sink for the inflowing $3 \mathrm{He}$, reducing the cooling requirements at the $4-\mathrm{K}$ stage.

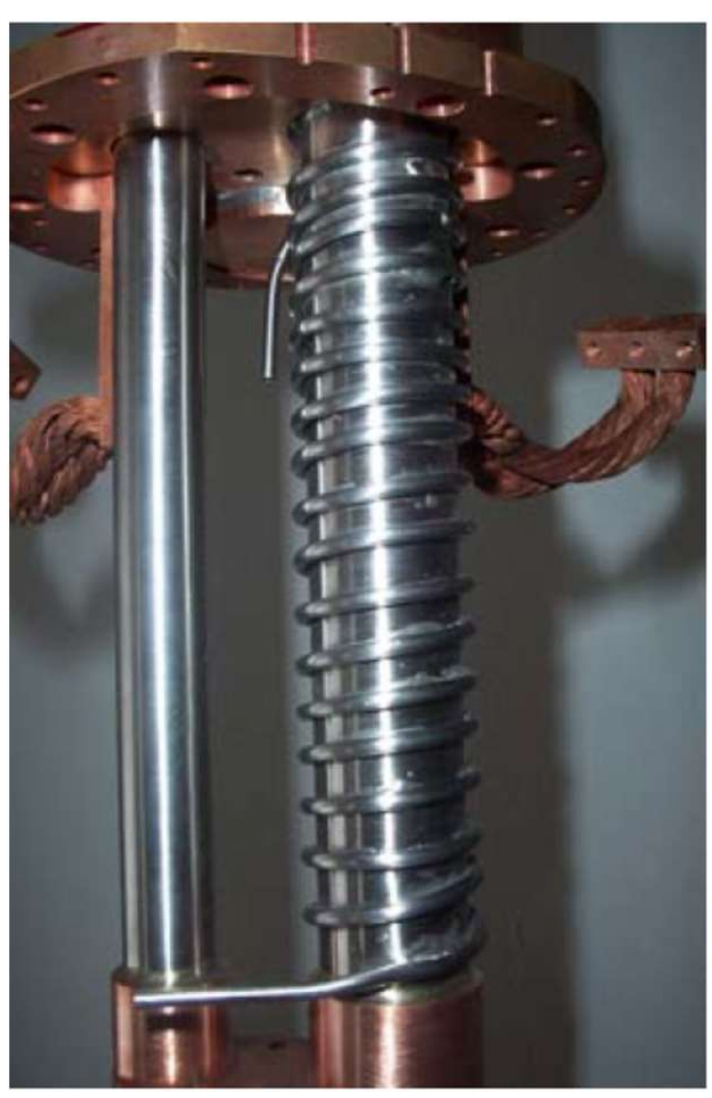

Additional heat exchanger (Uhlig, personal communication)

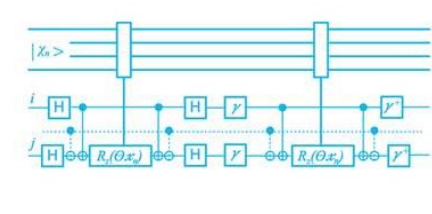




\section{Modern Dilution Refrigerator Design and Operation}




\section{Modern refrigerator layout}

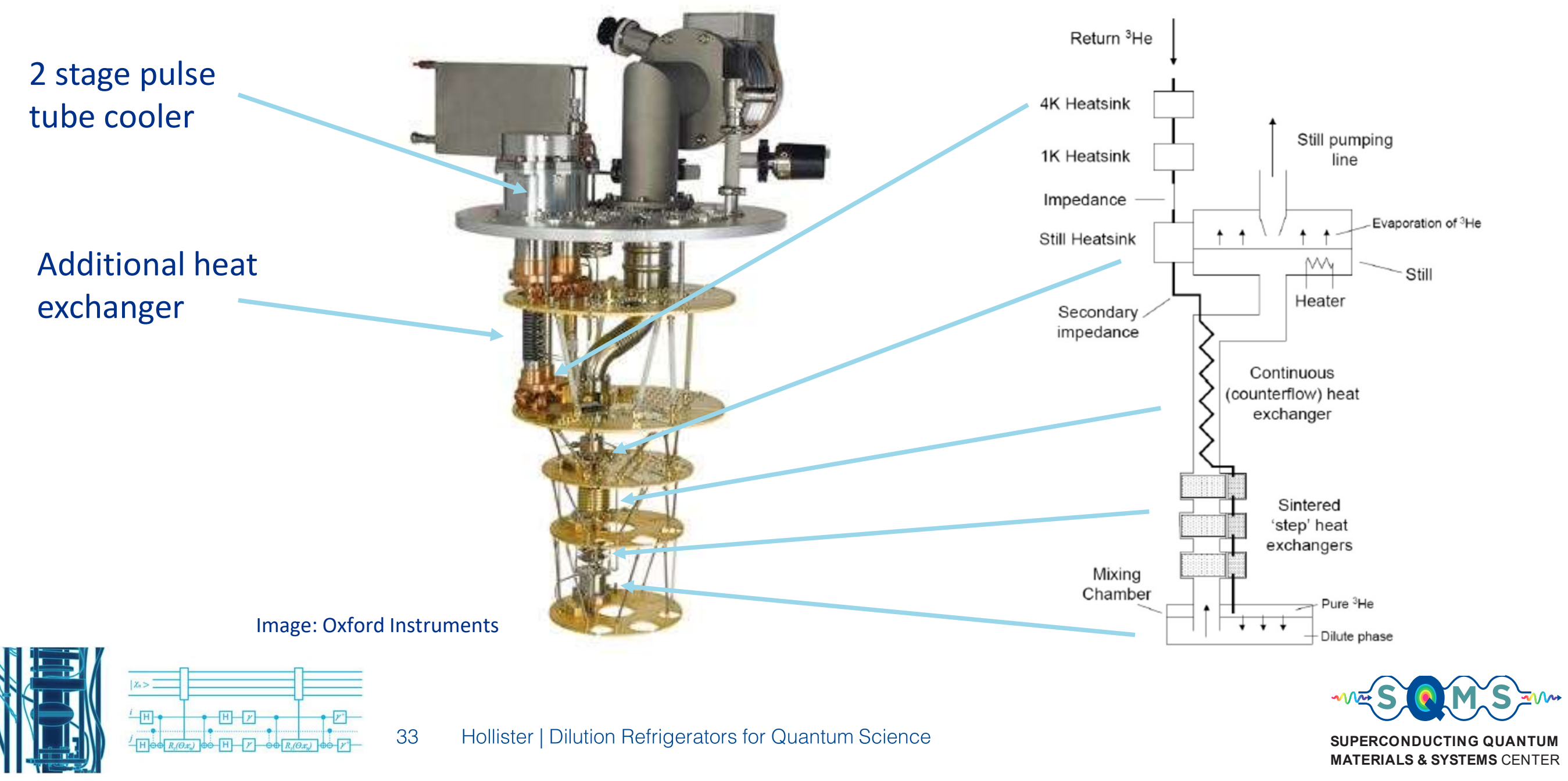




\section{Modern refrigerator layout - temperature stages and dilution unit}

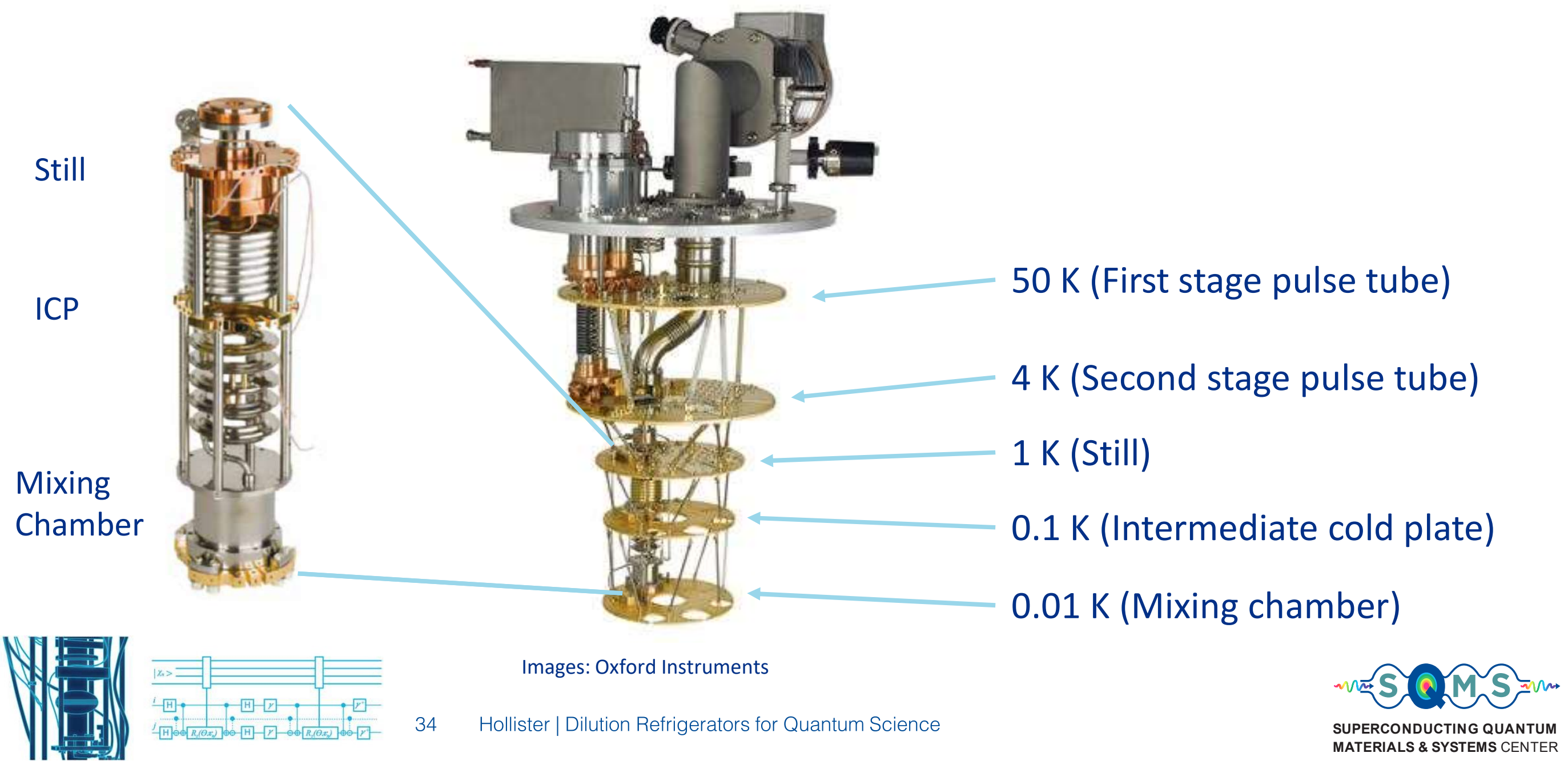




\section{Commercial fridge examples (1)}

Image: Oxford Instruments

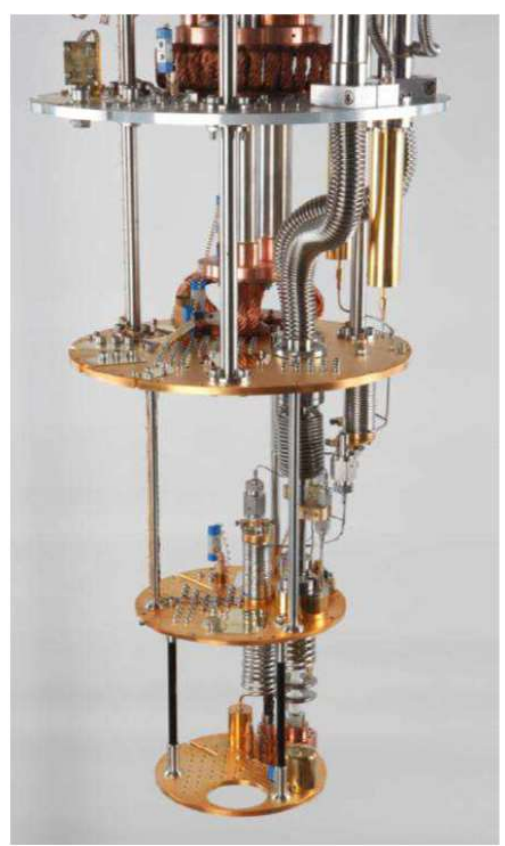

Small fridges

Mixing chamber diameter $\sim 150 \mathrm{~mm}$

Minimum temperature $\sim 25 \mathrm{mK}$

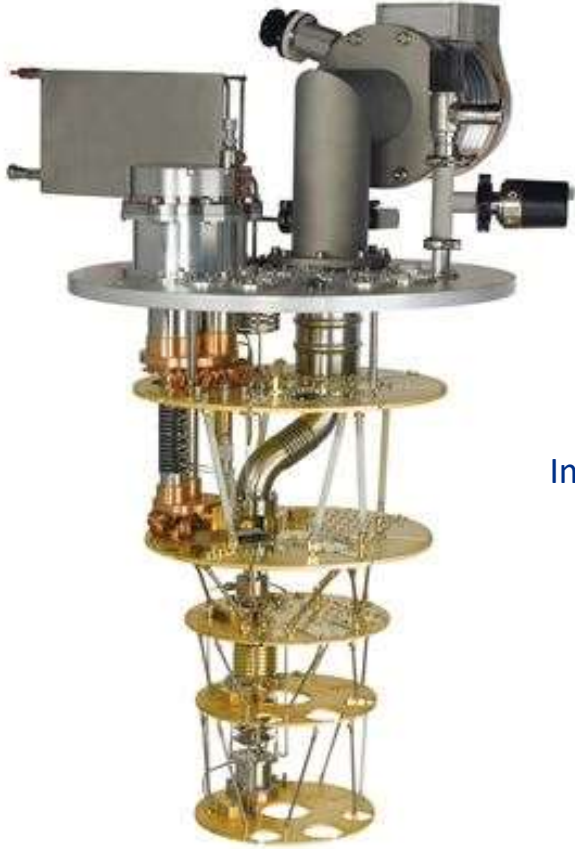

Image: Oxford Instruments

Standard fridges

Mixing chamber diameter $\sim 300 \mathrm{~mm}$

Minimum temperature $\sim 10 \mathrm{mK}$

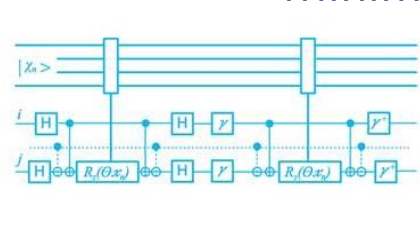




\section{Commercial fridge examples (2)}

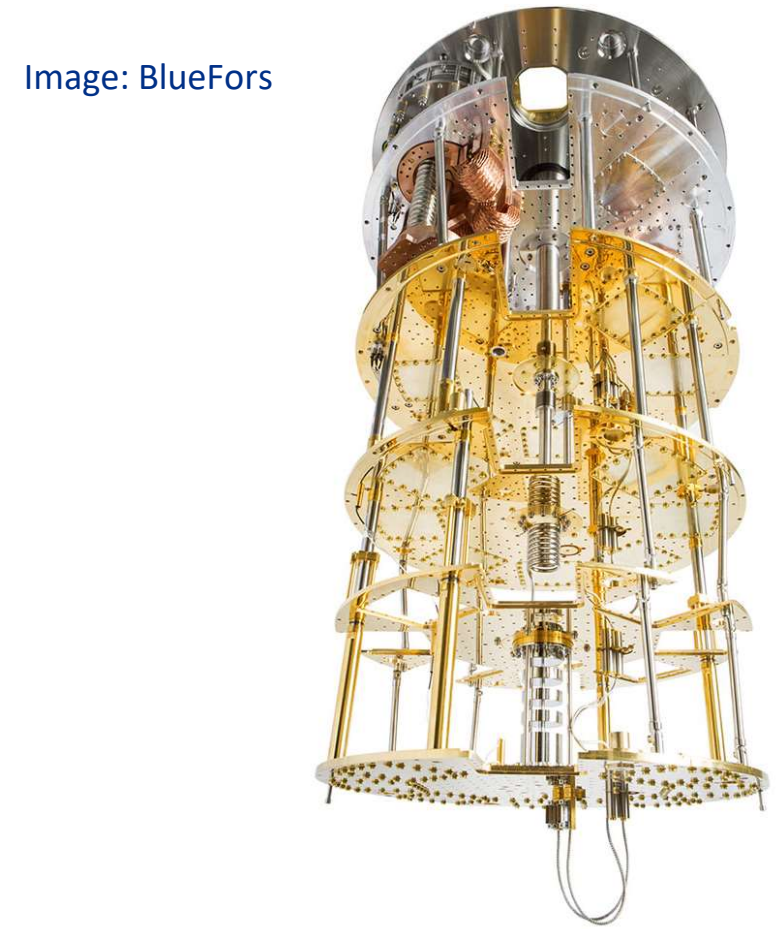

Large frame fridges

Mixing chamber diameter $\sim 500 \mathrm{~mm}$

Minimum temperature $\sim 10 \mathrm{mK}$

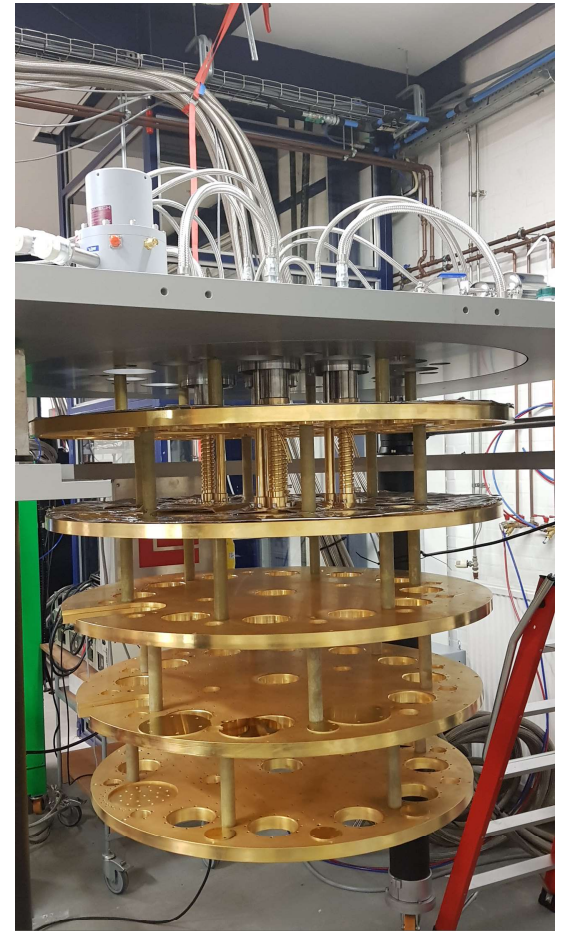

"XXL" fridges

Image: Leiden Cryogenics 


\section{Operational Considerations (1)}

Symptom: High condensing pressure

Possible causes:

1. Blockage in the input lines

2. Blockage in the cold trap

3. High temperature on the condensing stages, either the 4-K stage or the Still

Symptom: Slow cooling, particularly during condensation

Possible causes:

1. Large heat capacity

2. Poor thermal connection 


\section{Operational Considerations (2)}

Symptom: Low circulation rate/pressure

Possible causes:

1. Low still temperature

2. If combined with high still temperature, possibly too much or too little mixture

Symptom: High mixing chamber temperature

Possible causes:

1. Heat leak or thermal short

2. If combined with weak response to heat input, Quantity of Helium-3 may be too low (phase boundary is not in the mixing chamber). 


\section{Operational Considerations (3)}

- Some general operational comments:

- Monitor the system behavior run-to-run. Differences in temperatures, pressures and performance can be useful indicators of problems.

- Monitor how much mixture you are recovering at the end of the cool down, useful to monitor for leaks.

- Be aware of how much contamination is accumulating in the cold traps between runs since this can indicate the presence of an air leak into the system.

- Be aware of recommended maintenance internals on pumps. Can be a source of performance issues or leaks. 


\section{Future Developments}




\section{Larger and more powerful fridges}

- Drive has been towards larger cold volumes and higher cooling power to accommodate more wires and cryogenic components.

- Recently, 1-meter class fridges have started to come on the market.

- Are Fermilab, we are constructing a 2-meter refrigerator.

- Higher cooling power is challenging due to the need to make larger heat exchangers.

- This can be overcome by making several dilution circuits operating in parallel to increase the available cooling on a common mixing chamber plate.

- Issues with cooling power at the higher temperature stages could be addressed by using liquid cryogens rather than closed-cycle coolers. Not practical for small labs, but for large centers with multiple platforms having a liquid helium plant becomes viable. 


\section{Summary, Concluding Remarks, References}




\section{Summary}

- We have reviewed:

- The physics or Helium-3/Helium-4 mixtures and the dilution process

- Practical aspects of constructing a refrigerator and the design of key components

- The history of development of modern, cryogen-free refrigerators

- Current state-of-the-art

- Operational considerations and trouble shooting for common problems

- Possible future developments 


\section{Final Remarks}

- Thank you for your attention. Hopefully, you've found this to be useful.

- This is a very in-depth subject, and we've only taken a very brief tour through the processes and engineering associated with these machines.

- Theoretical knowledge is great, but there's no substitute for practical experience!

- If anyone has questions or would like to discuss any of these points further, please reach out to me during the conference via Whova. 


\section{Useful References}

- "Matter and Methods at Low Temperatures", Frank Pobell - Very good general textbook for low temperature physics

- "Experimental Principles and Methods Below 1K", O.V. Lounasmaa - Good description of the dilution refrigeration process, although was published in 1974 so is out of date on the technology

- "Experimental Techniques for Low-Temperature Measurements", Jack Ekin - Not so much about the cooling techniques themselves, but has a wealth of cryogenic reference data 


\section{Commercial Manufacturers of Dilution Refrigerators}

Provided for reference only. Not necessarily complete, and certainly not an endorsement of products from any one vendor.

- BlueFors Cryogenics (https://bluefors.com)

- Chase Research Cryogenics (https://www.chasecryogenics.com)

- CryoConcepts (https://cryoconcepts.com)

- Entropy Cryogenics (https://www.entropy-cryogenics.com)

- Innovative Cryogenic Engineering (https://www.iceoxford.com)

- Janis ULT (https://www.janult.com)

- Leiden Cryogenics (https://leiden-cryogenics.com)

- Oxford Instruments (https://www.oxinst.com) 


\section{Acknowledgement}

This manuscript has been authored by Fermi Research Alliance, LLC under Contract No. DE-AC02$07 \mathrm{CH} 11359$ with the U.S. Department of Energy, Office of Science, Office of High Energy Physics. 